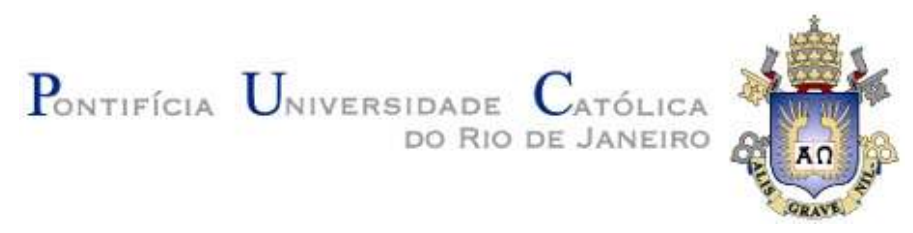

Felipe Andrade Oliveira

\title{
Risco Percebido e Orientação para Compras: Um Estudo Empírico em Varejo Multicanal
}

Dissertação de Mestrado

Dissertação apresentada ao Programa de PósGraduação em Administração de Empresas da PUCRio como requisito parcial para obtenção do título de Mestre em Administração de Empresas.

Orientador: Prof. Luis Fernando Hor-Meyll Alvares

Rio de Janeiro Dezembro de 2009 


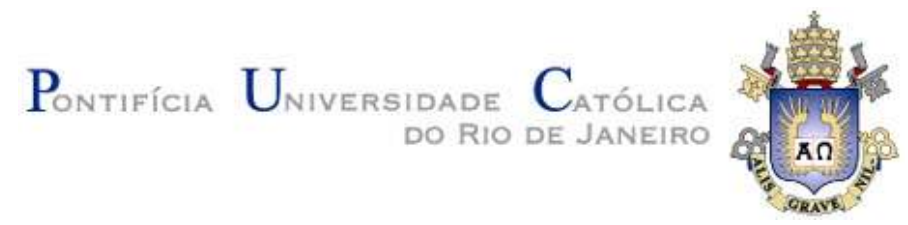

Felipe Andrade Oliveira

\section{Risco Percebido e Orientação para Compras: Um Estudo Empírico em Varejo Multicanal}

Dissertação apresentada como requisito parcial para obtenção do grau de Mestre pelo Programa de PósGraduação em Administração da PUC-Rio. Aprovada pela Comissão Examinadora abaixo assinada.

Prof. Luis Fernando Hor-Meyll Alvares Orientador Departamento de Administração - PUC-Rio

Prof. Paulo Cesar de Mendonça Motta Departamento de Administração - PUC-Rio

Prof. Valdecy Faria Leite Universidade Federal do Rio de Janeiro - UFRJ

Rio de Janeiro, 17 de dezembro de 2009. 
Todos os direitos reservados. É proibida a reprodução total ou parcial do trabalho sem autorização da universidade, do autor e do orientador.

\section{Felipe Andrade Oliveira}

Graduou-se em Engenharia Civil na Universidade Federal do Rio de Janeiro em 2006. Dedicou-se parcialmente ao Mestrado em Administração de Empresas. Trabalha há três anos no mercado de varejo atuando primeiramente no canal físico e, a partir deste ano, atua no canal on-line.

Ficha Catalográfica

Oliveira, Felipe Andrade

Risco percebido e orientação para compras ; um estudo empírico em varejo multicanal / Felipe Andrade Oliveria ; orientador: Luis Fernando Hor-Meyll Alvares. - 2009.

97 f. ; $30 \mathrm{~cm}$

Dissertação (Mestrado em Administração)-Pontifícia Universidade Católica do Rio de Janeiro, Rio de Janeiro, 2009.

Inclui bibliografia

1. Administração - Teses. 2. Risco percebido. 3. Orientação para compras. 4. Comportamento do consumidor. 5. Varejo multicanal. I. Alvares, Luis Fernando Hor-Meyll. II. Pontifícia Universidade Católica do Rio de Janeiro. Departamento de Administração. III. Título. 
Aos meus pais, Marcos e Maria Célia. 


\section{Agradecimentos}

A Deus, por te me dado a oportunidade de ter chegado até aqui.

Aos meus pais, Marcos e Maria Célia, por terem montado uma família de muito valor, por me fazerem forte para superar as adversidades da vida e pelos incansáveis suportes nos momentos difíceis.

Aos meus familiares: meu avô José, pela amizade e companheirismo; minha irmã Flávia, meus tios Vera Laís e Cid, pela torcida e apoio incondicionais.

Ao meu amigo e orientador Luis Fernando, essencial na realização deste trabalho de pesquisa, pelo apoio e orientação.

Aos professores Silvia Zaharia, pelo auxílio na elaboração do questionário, e Marcelo Cabús, pelo apoio na análise dos dados.

A todos os professores e funcionários do IAG/PUC-Rio, pela excelência na passagem dos diversos ensinamentos, em especial a Teresa Campos e Fábio Etienne.

A todos os professores e pessoas envolvidas no meu intercâmbio na Universidade Católica Portuguesa em Lisboa (Portugal), pela excelência na infra-estrutura e passagem dos diversos ensinamentos.

À empresa pesquisada, fonte do estudo de caso, pela oportunidade de realizar a pesquisa.

Aos meus amigos de longa data, pelos diversos momentos de alegria vividos.

A todos os meus amigos do trabalho, por tornarem o meu dia-a-dia melhor e mais divertido.

A todos os meus amigos do IAG/PUC-Rio, pela forte amizade criada. 
Agradeço também a algumas pessoas especiais que contribuíram de certa forma para a elaboração deste trabalho: Andréia Moraes, Camila Bezerra, Camila Borges, Carlos Rezende, Cristiane Santos, Eduardo Vaz, Fabiene Costa, Fábio Pacheco, Guilherme D’Aguiar, Joana Fonseca, Juliana Verleum, Kátia Martins, Luis Roberto Pinto, Mariana Lemgruber, Mariana Tavares, Marina Neves, Nágia Pinto, Paula Salinas, Rafael Fernandes, Renata Pinto, Rodrigo Otávio. 


\section{Resumo}

Oliveira, Felipe Andrade. Alvares, Luis Fernando Hor-Meyll. Risco Percebido e Orientação para Compras: Um Estudo Empírico em Varejo Multicanal. Rio de Janeiro, 2009. 97p. Dissertação Mestrado - Departamento de Administração. Pontifícia Universidade Católica do Rio de Janeiro.

Diversos estudos sobre risco percebido em compras online têm sido realizados, porém poucas foram as pesquisas que compararam a percepção de risco do consumidor entre canais distintos de uma mesma empresa de varejo. Da mesma forma, também são poucos os estudos encontrados na literatura que consideram o efeito da orientação para compras sobre a propensão do consumidor realizar compras online. Nesta pesquisa, conduziu-se um survey em uma amostra de 397 consumidores de um grande varejista multicanal, para mensurar a percepção de risco e a orientação de compra predominante em decisões de compra que privilegiam um dos canais disponibilizados pela empresa - Internet ou suas lojas físicas. Os resultados obtidos sugerem que o risco de desempenho e a orientação por conveniência são predominantes para a escolha do canal utilizado para a busca de informações, enquanto que os riscos de tempo, psicológico e financeiro são relevantes para a escolha, pelo consumidor, do canal em que será efetuada a transação de compra. Os consumidores que buscam informações no canal online, mas efetivam a compra na loja física, parecem perceber risco financeiro e risco de tempo (ou de conveniência) mais elevados do que os consumidores que privilegiam a loja online para essas duas etapas do processo de compra. Os consumidores que buscam informações na loja física, mas preferem efetuar a transação na loja online, parecem perceber maior risco de desempenho, sendo orientados mais por recreação do que os consumidores que tanto buscam informações quanto efetuam a transação no canal online.

\section{Palavras-Chave}

Risco Percebido, Orientação para Compras; Comportamento do Consumidor; Varejo Multicanal. 


\section{Abstract}

Oliveira, Felipe Andrade. Alvares, Luis Fernando Hor-Meyll. Perceived risk and Shopping Orientation: An Empirical Study in Multichannel Retail. Rio de Janeiro, 2009. 97p. MSc Dissertation - Departamento de Administração. Pontifícia Universidade Católica do Rio de Janeiro.

Many studies on perceived risk in online shopping has been made, but few of them compared the consumer's risk perception between different channels of the same multichannel company. Similarly, there are few studies in literature that consider the effect of shopping orientation on the propensity of consumers to make purchases online. In this study, a survey was performed in a sample of 397 consumers of a large multichannel retailer, to measure the predominant risk perception and purchase orientation in purchasing decisions that decide the choice of one of the channels offered by the company - Internet or their physical stores.

The results suggest that the performance risk and convenience orientation are predominant in the choice of channel used for information search, while the time, psychological and financial risks are relevant to make a purchase. Consumers who seek information on the online store but buy in physical store seem to perceive higher financial and time risks (or convenience) than those consumers who prefer to use the online channel for these two buying process stages. Consumers who seek information on the physical store prefer to complete the transaction online store seem to realize higher performance risk and are more recreational oriented than consumers who seek information and buy the product on the online store.

\section{Keywords}

Perceived Risk, Shopping Orientation, Consumer Behavior, Multichannel Retail. 


\section{Sumário}

Resumo

1 Descrição do Problema 14

1.1. Introdução 14

1.1.1. Desenvolvimento da Internet no Brasil 15

1.1.2. Os obstáculos encontrados pelo E-commerce 16

1.1.3. O atual cenário do E-commerce no Brasil 17

1.1.4. Algumas características do consumidor do E-commerce no Brasil 18

$\begin{array}{ll}\text { 1.2. Objetivo } & 21\end{array}$

1.3. Relevância $\quad 21$

1.4. Delimitações do Estudo 22

1.5. Estrutura da Dissertação 23

2 Revisão da Literatura $\quad 24$

2.1. Os Conceitos de Risco Percebido 24

2.2. A importância do Risco Percebido no processo decisório de compra 26

2.2.1. O processo decisório de compra 26

2.3. As Dimensões de Risco Percebido $\quad 27$

$\begin{array}{lr}\text { 2.3.1. Risco Financeiro } & 27\end{array}$

$\begin{array}{ll}\text { 2.3.2. Risco de Desempenho } & 28\end{array}$

$\begin{array}{lr}\text { 2.3.3. Risco Físico } & 28\end{array}$

$\begin{array}{ll}\text { 2.3.4. Risco Psicossocial } & 28\end{array}$

2.3.5. Risco de Tempo 29

2.4. Risco Inerente e Risco Manipulado 29

2.5. O Risco Percebido e o E-commerce 30 
2.5.1 A confiança do consumidor no canal de compra on-line

2.5.2. As Dimensões de Risco Percebido e o E-commerce 31

2.5.3. A confiança no varejista on-line 32

2.6. A Orientação para Compras 33

2.7. O Varejo Multicanal 34

2.7.1. A influência do Varejo Multicanal no processo decisório de compra 36

$\begin{array}{ll}\text { 2.8. Formulação das hipóteses substantivas } & 37\end{array}$

3 Procedimentos Metodológicos $\quad 42$

3.1. Tipo de Pesquisa 42

3.2. Universo da amostra $\quad 42$

$\begin{array}{ll}3.2 .1 \text { Amostra } & 43\end{array}$

3.3. Operacionalização das variáveis 44

3.3.1. Variáveis relacionadas ao constructo Risco Percebido 44

3.3.2. Variáveis relacionadas à Orientação para compras 44

3.3.3. Variáveis relacionadas aos canais de compra 45

$\begin{array}{ll}\text { 3.3.4. As variáveis demográficas } & 46\end{array}$

3.4. Construção do Instrumento de Medida 46

3.4.1. Construção do Questionário 46

3.4.1.1. Escala para mensurar os canais de compra utilizados 47

3.4.1.4. Escalas para as variáveis demográficas 48

3.4.2. Definição operacional das variáveis 49

3.5. Coleta de dados 50

3.6. Limitações do método $\quad 50$

4 Análise de Dados $\quad 51$

4.1. Perfil dos Respondentes $\quad 51$

4.2. Análise dos dados $\quad 54$

4.3. Análise das Dimensões de Risco Percebido e Orientação para compras 56 
4.3.1. Análise Fatorial do constructo risco percebido

4.3.2. Análise Fatorial da Orientação para Compras

4.3.3. Teste de confiabilidade da escala utilizada

4.3.4. Análise das variáveis que diferenciam os grupos - Análise Discriminante60

4.3.5. Teste das hipóteses

61

4.3.5.1. Teste das hipóteses $\mathrm{H} 1$ e $\mathrm{H} 2$

62

4.3.5.2. Teste da hipótese H3

63

4.3.5.3. Teste das hipóteses $\mathrm{H} 4$ e H5

64

4.3.5.4. Teste das hipóteses $\mathrm{H} 6$ e H7

66

4.3.5.5. Teste das hipóteses $\mathrm{H} 8$ e H9

68

5 Conclusões e Recomendações

70

5.1. Conclusão

70

5.2. Resposta à primeira pergunta

71

5.3. Resposta à segunda pergunta

72

5.4. Recomendações e estudos futuros

74

6 Referências Bibliográficas

76

7 Anexos

85 


\section{Lista de Tabelas}

TABELA 1: QUANTIDADE DE E-CONSUMIDORES NO BRASIL $\quad 17$

TABELA 2: PROdUtOS MAIS VENDIDOS NO VAREJO ON-LINE DO BRASIL 19

TABELA 3: OPÇÕES POSSÍVEIS PARA BUSCA DE INFORMAÇÕES E COMPRA. 37

TABELA 4: COMBINAÇÃO DE CANAIS UTILIZADOS NA PESQUISA

TABELA 5: MÉdIA E DESVIOS PADRÃO DO QUESTIONÁRIO 55

TABela 6: Teste de KMO e Bartlett PaRA RISCo PeRCEBIdo 56

TABELA 7: MATRIX DE FATORES PARA RISCO PERCEBIDO 57

TABela 8: Teste de KMO E BARTLetT PARA ORIENTAÇÃo PARA Compras 58

TABELA 9: MATRIX DE FATORES PARA ORIENTAÇÃO PARA COMPRAS 58

TABELA 10: CÁlCULO do ALFA DE CRONBACH POR GRUPOS DE ENTREVISTADOS 59

TABELA 11: RESULTADOS DA ANÁLISE DESCRIMINANTE 60

TABELA 12: MÉDIAS E DESVIOS PADRÕES DE ORE E OCO PARA FI+FI E ON+ON 62

TABELA 13: TESTE DE AMOSTRAS - VARIÁVEL ORE E FI+FI E ON+ON 62

TABELA 14: TESTE DE AMOSTRAS - VARIÁVEL OCO E FI+FI E ON+ON 63

TABELA 15: MÉDIAS E DESVIOS PADRÕES DE OCO PARA FI+FI E ON+FI 64

TABELA 16: TESTE DE AMOSTRAS - VARIÁVEL OCO E ON+FI E FI+FI 64

TABELA 17: MÉdIAS E DESVIOS PADRÕES DE RF E RT PARA ON+FI E ON+ON. 65

TABELA 18: TESTE DE AMOSTRAS - VARIÁVEL RF E ON+FI E ON+ON 65

TABELA 19: TESTE DE AMOSTRAS - VARIÁVEL RT E ON+FI E ON+ON 65

TABELA 20: MÉDIAS E DESVIOS PADRÕES DE RD E ORE PARA FI+ON E ON+ON. 66

TABELA 21: TESTE DE AMOSTRAS - VARIÁVEL RD E FI+ON E ON+ON 67 
TABELA 22: TESTE DE AMOSTRAS - VARIÁVEL ORE E FI+ON E ON+ON

TABELA 23: MÉDIAS E DESVIOS PADRÕES DE RF E RT PARA FI+ON E FI+FI

TABELA 24: TESTE DE AMOSTRAS - VARIÁVEL RF E FI+ON E FI+FI

68

TABELA 25: TESTE DE AMOSTRAS - VARIÁVEL RT E FI+ON E FI+FI

69

\section{Lista de Gráficos}

GRÁFICO 1: FATURAMENTO ANUAL DO VAREJO ON-LINE (EM BILHÕES) 18

GRÁFICO 2: RENDA FAMILIAR MENSAL DO CONSUMIDOR DA INTERNET 43

GRÁFICO 3: FAIXA ETÁRIA E ESCOLARIDADE DO CONSUMIDOR DA INTERNET

43

GRÁFICO 4: PREFERÊNCIA POR CANAL UTILIZADO PARA INFORMAÇÕES E COMPRAS 52

GRÁFICO 5: DISTRIBUIÇÃO POR GÊNERO DO RESPONDENTE

GRÁFICO 6: DISTRIBUIÇÃO POR FAIXA ETÁRIA DO RESPONDENTE 53

GRÁFICO 7: DISTRIBUIÇÃO POR ESCOLARIDADE E POR RENDA FAMILIAR MENSAL 53 